Bull. Korean Math. Soc. 50 (2013), No. 2, pp. 469-473

http://dx.doi.org/10.4134/BKMS.2013.50.2.469

\title{
A CORRECTION TO A PAPER ON ROMAN $k$-DOMINATION IN GRAPHS
}

\author{
Doost Ali Mojdeh* And Seyed Mehdi Hosseini Moghaddam
}

\begin{abstract}
Let $G=(V, E)$ be a graph and $k$ be a positive integer. A $k$ dominating set of $G$ is a subset $S \subseteq V$ such that each vertex in $V \backslash S$ has at least $k$ neighbors in $S$. A Roman $k$-dominating function on $G$ is a function $f: V \rightarrow\{0,1,2\}$ such that every vertex $v$ with $f(v)=0$ is adjacent to at least $k$ vertices $v_{1}, v_{2}, \ldots, v_{k}$ with $f\left(v_{i}\right)=2$ for $i=1,2, \ldots, k$. In the paper titled "Roman $k$-domination in graphs" (J. Korean Math. Soc. 46 (2009), no. 6, 1309-1318) K. Kammerling and L. Volkmann showed that for any graph $G$ with $n$ vertices, $\gamma_{k R}(G)+\gamma_{k R}(\bar{G}) \geq \min \{2 n, 4 k+1\}$, and the equality holds if and only if $n \leq 2 k$ or $k \geq 2$ and $n=2 k+1$ or $k=1$ and $G$ or $\bar{G}$ has a vertex of degree $n-1$ and its complement has a vertex of degree $n-2$. In this paper we find a counterexample of Kammerling and Volkmann's result and then give a correction to the result.
\end{abstract}

\section{Introduction}

Let $G=(V, E)$ be a graph with vertex set $V=V(G)$ and edge set $E=E(G)$. A $k$-dominating set of $G$ is a subset $S \subseteq V$ such that every vertex in $V \backslash S$ has at least $k$ neighbors in $S$. The $k$-domination number $\gamma_{k}(G)$ of $G$ is the minimum cardinality among the $k$-dominating sets of $G$. A 1-domination number $\gamma_{1}(G)$ is identified with the usual domination number $\gamma(G)$ (see $[1,3,5]$ ). A Roman $k$-dominating function on a graph $G$ is a function $f: V \rightarrow\{0,1,2\}$ such that every vertex $v$ with $f(v)=0$ is adjacent to at least $k$ vertices $v_{1}, v_{2}, \ldots, v_{k}$ with $f\left(v_{i}\right)=2$ for $i=1,2, \ldots, k$. The weight of a Roman $k$-dominating function $f$ is the value $f(V)=\sum_{u \in V} f(u)$. The minimum weight of a Roman $k$-dominating function on a graph $G$ is said to be the Roman k-domination number $\gamma_{k R}(G)$ of $G$. A Roman $k$-dominating function on a graph $G$ of minimum weight is called a $\gamma_{k R}$-function of $G$. A Roman 1-domination number $\gamma_{1 R}(G)$ of a graph $G$ is identified with the usual Roman domination number $\gamma_{R}(G)$ (see $[2,4]$ ). The

Received October 22, 2011; Revised April 7, 2012

2010 Mathematics Subject Classification. 05C69.

Key words and phrases. dominating set, Roman $k$-dominating function, correction.

*This research was in part supported by a grant from IPM (No. 90050045). 
order of a graph $G=(V, E)$ is the cardinality of $V$ denoted by $|V|$ or $n(G)$ and the induced subgraph of $G$ generated by subset $U \subseteq V$ is denoted by $G[U]$.

In 2009, K. Kammerling and L. Volkmann [2] studied Roman $k$-domination number of graphs and they showed the following.

Theorem 1 ([2], Theorem 2.8). If $G$ is a graph of order $n$, then

$$
\gamma_{k R}(G)+\gamma_{k R}(\bar{G}) \geq \min \{2 n, 4 k+1\} .
$$

Furthermore the equality holds in (1) if and only if $n \leq 2 k$ or $k \geq 2$ and $n=2 k+1$ or $k=1$ and $G$ or $\bar{G}$ has a vertex of degree $n-1$ and its complement has a vertex of degree $n-2$.

In this paper, we find a counterexample of the equality part of the above result and then give a correction to this result.

\section{Main results}

In this section we improve Theorem 1 . The following results from [2] are useful.

Theorem 2 ([2], Proposition 2.6). If $G$ is a graph of order $n$, then $\gamma_{k R}(G) \geq$ $\min \left\{n, \gamma_{k}(G)+k\right\}$.

Theorem 3 ([2], Proposition 2.7). Let $G$ be a graph of order $n$.

(i) If $n \leq 2 k$, then $\gamma_{k R}(G)=n$.

(ii) If $n \geq 2 k+1$, then $\gamma_{k R}(G) \geq 2 k$.

(iii) If $n \geq 2 k+1$ and $\gamma_{k}(G)=k$, then $\gamma_{k R}(G)=\gamma_{k}(G)+k=2 k$.

The following has a straightforward proof, so its proof is left to the reader.

Observation 4. Let $G$ be a graph with $t$ component $H_{1}, H_{2}, \ldots, H_{t}$. Then

$$
\gamma_{k R}(G)=\sum_{i=1}^{t} \gamma_{k R}\left(H_{i}\right)
$$

First we present a counterexample.

A counterexample to Theorem 1. Let $k$ be a positive integer $k \geq 2$, and let $G$ be a graph such that $V(G)=\left\{a_{0}, a_{1}, a_{2}, \ldots, a_{2 k}\right\}, E(G)=\left\{a_{0} a_{i} \mid 1 \leq\right.$ $i \leq k\} \cup\left\{a_{2 i-1} a_{2 i} \mid 1 \leq i \leq k\right\}$ (see Figure 1 for an illustration).

It is easy to see that $\gamma_{k R}(G) \leq 2 k+1$ and $\gamma_{k R}(\bar{G}) \leq 2 k+1$, since the function defined by $f(v)=1$ for all $v$ is a Roman $k$-dominating function on both $G$ and $\bar{G}$. We will show that $\gamma_{k}(G)>k$. Suppose that there exists a $k$-dominating set $D$ of $G$ such that $|D|=k$. Then any vertex in $D$ is adjacent to any vertex in $V(G) \backslash D$. Since $|V(G)|=2 k+1$ and $|D|=k, G$ has $k$ vertices whose degrees are at least $k+1$. However, the vertex $a_{0}$ is the only one vertex which has degree at least $k+1$, a contradiction. Therefore, $\gamma_{k}(G)>k$ and so $\gamma_{k R}(G) \geq 2 k+1$ by Theorem 2 . We can conclude that $\gamma_{k R}(G)=2 k+1$.

Now consider the complement $\bar{G}$ of $G$. Then $\bar{G}$ is the disjoint union of an isolated vertex $a_{0}$ and the complete $k$-partite graph with partite sets of equal 


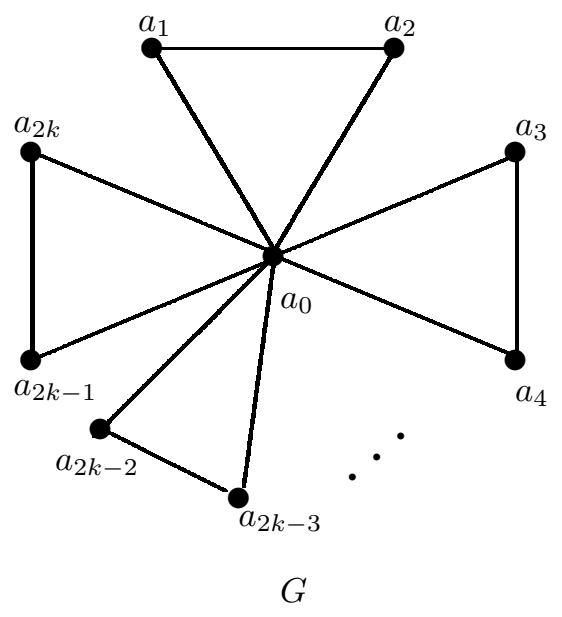

Figure 1.

size 2, and call those two connected components $H_{1}$ and $H_{2}$, respectively. By (i) of Theorem 3, $\gamma_{k R}\left(H_{1}\right)=1$ and $\gamma_{k R}\left(H_{2}\right)=2 k$. By Observation 4, $\gamma_{k R}(\bar{G})=\gamma_{k R}\left(H_{1}\right)+\gamma_{k R}\left(H_{2}\right)$, and therefore $\gamma_{k R}(\bar{G})=2 k+1$.

As we shown that $\gamma_{k R}(G)=\gamma_{k R}(\bar{G})=2 k+1$, we obtain that

$$
\gamma_{k R}(G)+\gamma_{k R}(\bar{G})=4 k+2>\min \{2|V(G)|, 4 k+1\}=4 k+1,
$$

which violates the equality part of Theorem 1 .

Now we give a correction of Theorem 1 . If $f: V \rightarrow\{0,1,2\}$ is a Roman $k$-dominating function on a graph $G$, then $\left\{V_{0}, V_{1}, V_{2}\right\}$ is a partition of $V$ where for $i=0,1,2, V_{i}=\{v \in V(G) \mid f(v)=i\}$. In the rest of the paper, we denote the function $f$ by $\left(V_{0}, V_{1}, V_{2}\right)$ for simplicity.

Theorem 5. If $G$ is a graph of order $n$, then $\gamma_{k R}(G)+\gamma_{k R}(\bar{G}) \geq \min \{2 n, 4 k+$ $1\}$ and the equality holds if and only if one of the following holds:

(i) $n \leq 2 k$;

(ii) $n=2 k+1$, and either $\gamma_{k}(G)=k$ or $\gamma_{k}(\bar{G})=k$;

(iii) $k=1, n \geq 4$ and $G$ or $\bar{G}$ has a vertex of degree $n-1$ and its complement has a vertex of degree $n-2$.

Proof. The proof of inequality part is identified with the correspondence proof of Theorem 1 ([2] Theorem 2.8).

If (i) holds, then $\gamma_{k R}(G)=n=\gamma_{k R}(\bar{G})$ and $\gamma_{k R}(G)+\gamma_{k R}(\bar{G})=2 n$ and therefore $2 n=\min \{2 n, 4 k+1\}$.

Suppose that (ii) holds. Without loss of generality, we assume that $\gamma_{k}(G)=$ $k$. By (iii) of Theorem $2, \gamma_{k R}(G)=2 k$. Since $f(\emptyset, V(\bar{G}), \emptyset)$ is a $\gamma_{k R}$-function of $\bar{G}, \gamma_{k R}(\bar{G}) \leq n=2 k+1$. Therefore $\gamma_{k R}(G)+\gamma_{k R}(\bar{G}) \leq 4 k+1$. From the inequality part and the fact that $\min \{2 n, 4 k+1\}=4 k+1$, it holds that $\gamma_{k R}(G)+\gamma_{k R}(\bar{G}) \geq 4 k+1$. Thus $\gamma_{k R}(G)+\gamma_{k R}(\bar{G})=4 k+1$. 
Let $k=1, n \geq 4$ and $G$ or $\bar{G}$ has a vertex of degree $n-1$ and its complement has a vertex of degree $n-2$. We can assume that $G$ has a vertex of degree $n-1$. Therefore there exists a vertex in $G$ that dominates $G$ and hence $\gamma_{k R}(G)=2$. The vertex of degree $n-1$ is an isolated vertex in $\bar{G}$. Thus the isolated vertex and the vertex of degree $n-2$ in $\bar{G}$ dominate $\bar{G}$. So $\gamma_{k R}(\bar{G})=2+1=3$ and $\gamma_{k R}(G)+\gamma_{k R}(\bar{G})=\gamma_{R}(G)+\gamma_{R}(\bar{G})=2+3=5=\min \{2 n, 4 k+1\}$.

Conversely, let $\gamma_{k R}(G)+\gamma_{k R}(\bar{G})=\min \{2 n, 4 k+1\}$. If $n \leq 2 k$, then (i) immediately follows. Suppose that $n \geq 2 k+1$. Then $\min \{2 n, 4 k+1\}=$ $4 k+1$. By (ii) of Theorem $2, \gamma_{k R}(G) \geq 2 k$ and $\gamma_{k R}(\bar{G}) \geq 2 k$. Without loss of generality, we may assume that $\gamma_{k R}(G)=2 k$ and $\gamma_{k R}(\bar{G})=2 k+1$. Since $\gamma_{k R}(G)=2 k$, it follows that there exists a $\gamma_{k R}$-function $f\left(V_{0}, V_{1}, V_{2}\right)$ on $G$ such that $\left|V_{0}\right|=n-k, V_{1}=\emptyset,\left|V_{2}\right|=k$, and $V_{2}$ is a $k$-dominating set of $G$. Note that $\gamma_{k}(G)=k$.

Since any vertex of $V_{0}$ and any vertex of $V_{2}$ are adjacent in $G$ and $V_{1}=\emptyset, \bar{G}$ is the union of $\bar{G}\left[V_{0}\right]$ and $\bar{G}\left[V_{2}\right]$. Therefore, by Observation 4,

$$
\gamma_{k R}(\bar{G})=\gamma_{k R}\left(\bar{G}\left[V_{0}\right]\right)+\gamma_{k R}\left(\bar{G}\left[V_{2}\right]\right) .
$$

Since $\gamma_{k R}\left(\bar{G}\left[V_{2}\right]\right)=k$ by (i) of Theorem 2 and $\gamma_{k R}(\bar{G})=2 k+1$ by the assumption, it follows that $\gamma_{k R}\left(\bar{G}\left[V_{0}\right]\right)=k+1$.

On the other hand, since $\bar{G}\left[V_{0}\right]$ has $n-k$ vertices, by (i) and (ii) of Theorem 2, one of the following holds:

(a) $n-k \leq 2 k$ and $\gamma_{k R}\left(\bar{G}\left[V_{0}\right]\right)=n-k$;

(b) $n-k \geq 2 k+1$ and $\gamma_{k R}\left(\bar{G}\left[V_{0}\right]\right) \geq 2 k$.

Suppose that (a) holds. Then $k+1=n-k$ and so $n=2 k+1$. Since we already have $\gamma_{k}(G)=k$, (ii) immediately follows. Suppose that (b) holds. Then $k+1 \geq 2 k$ and so $k=1$. In addition, $n-k \geq 2 k+1$ implies $n \geq 4$. Since we already showed that any vertex in $V_{2}$ has degree $n-k, G$ has a vertex of degree $n-1$. Since $k=1, \gamma_{k R}\left(\bar{G}\left[V_{0}\right]\right)=k+1=2 k$, which implies that $\bar{G}\left[V_{0}\right]$ has a $k$-dominating set of size $k$. Then $\bar{G}\left[V_{0}\right]$ has a vertex which is adjacent to the other vertices of $\bar{G}\left[V_{0}\right]$. Since $\left|V_{0}\right|=n-k=n-1$, we can conclude that $\bar{G}$ has a vertex of degree $n-2$. Thus (iii) holds.

Acknowledgements. The authors would like to thank the referee for his/her very helpful comments and valuable suggestions.

\section{References}

[1] T. W. Haynes, S. T. Hedetniemi, and P. J. Slater, Fundamentals of Domination in Graphs, Marcel Dekker, New York, 1998.

[2] K. Kammerling and L. Volkmann, Roman k-domination in graphs, J. Korean Math. Soc. 46 (2009), no. 6, 1309-1318.

[3] C. S. Liao and G. J. Chang, Algorithmic aspect of $k$-tuple domination in graphs, Taiwanese J. Math. 6 (2002), no. 3, 415-420.

[4] C. S. ReVelle and K. E. Rosing, Defendens imperium Romanum: a classical problem in military strategy, Amer. Math. Monthly 107 (2000), no. 7, 585-594. 
[5] W. Shang, F. Yao, P. Wan, and X. Hu, On minimum $m$-connected $k$-dominating set problem in unit disc graphs, J. Comb. Optim. 16 (2008), no. 2, 99-106.

Doost Ali Mojdeh

Department of Mathematics

UNIVERSITY OF TAFRESH

TAFRESH, IRI

AND

SCHOOL OF MATHEMATiCS

Institute for Research in Fundamental Sciences (IPM)

Tehran, IRI, P.O. Box 19395-5746

E-mail address: damojdeh@ipm.ir

Seyed Mehdi Hosseini Moghaddam

Department of Mathematics

UnIVERSITY OF TAFRESH

TAFRESH, IRI

E-mail address: smehdi19@gmail.com 